\title{
Oralité / raison numérique. Circulation de la parole et des imaginaires
}

Nadine Decourt et Raymond Mayer

\section{OpenEdition}

\section{Journals}

Édition électronique

URL : http://journals.openedition.org/pa/178

DOI : $10.4000 /$ pa. 178

ISSN : 2273-0362

Éditeur

Université Lumière Lyon 2

\section{Édition imprimée}

Date de publication : 15 janvier 2009

Pagination : $37-45$

ISBN : 978-2-912868-62-6

ISSN : 1634-7706

Référence électronique

Nadine Decourt et Raymond Mayer, « Oralité / raison numérique. Circulation de la parole et des imaginaires », Parcours anthropologiques [En ligne], 7 | 2009, mis en ligne le 02 juillet 2013, consulté le 22 septembre 2020. URL : http://journals.openedition.org/pa/178; DOI : https://doi.org/10.4000/pa. 178 


\title{
Oralité / raison numérique Circulation de la parole et des imaginaires
}

\author{
Nadine Decourt \& Raymond Mayer
}

Le point commun de tous les articles de ce numéro est de tenter d'ouvrir une voie non "scripto-centrée » à la recherche sur l'oralité. Le débat habituel sur l'oralité se bâtit sur le préalable d'une " transcription " de l'oral. En associant ici l'oralité aux modalités du numérique, les auteurs voudraient fonder la recherche scientifique sur un nouveau « mariage de raison ». Tel est l'enjeu et telles sont les perspectives du présent numéro.

Dans un contexte où les productions scientifiques sont appelées à se compléter plutôt qu'à se répéter, quel intérêt y avait-il à consacrer ce numéro 7 des Parcours anthropologiques à deux thèmes déjà traités séparément dans des travaux de référence? On doit, en effet, à Françoise Waquet un ouvrage qui dispute explicitement de « la raison orale » (2003, 359-398), tandis qu'un numéro entier de la revue Hermès (2004, $\mathrm{n}^{\circ} 39$ ), coordonné par Virginie Paul et Jacques Perriault, est dévolu à la "critique de la raison numérique ". Qu'y avait-il à ajouter à ces deux contributions pionnières sur le sujet qui nous intéresse?

Il y avait à ajouter la confrontation directe entre les deux univers de " discours ", celui de l'oralité et celui de la numéricité, tant il est vrai que le second conduit à réinterroger le premier. Il n'est pas suffisant d'établir des constats, il faut en tirer les conséquences scientifiques. En avril 2006, à Lyon-2, un séminaire du CREA intitulé Raison orale, raison graphique, raison numérique a "débrouillé » la matière. Ce numéro voudrait en prolonger la manière. Il se présente donc comme un manifeste d'application d'un discours scientifique à un discours oral - lui-même positionné par rapport à un environnement numérique, dont on n'a que trop répété qu'il révolutionnait les idées, sans préciser lesquelles et par quels moyens. Ainsi défini, toutes les options étaient possibles. Les contributeurs de ce numéro se sont fait plaisir à diversifier leurs perspectives d'analyse, sans jamais chercher à décortiquer le sujet à la manière d'une entomologie. Le résultat est un vivant dialogue d'articles qui se répondent en variant les points de vue théoriques, les terrains d'observation et les considérations de synthèse.

L'oralité est un terme commode où se reconnaissent tant bien que mal des "militants de la parole", pour reprendre une expression de Philippe Breton dans son Éloge de la parole (2007 : 9, 20-23), venus d'horizons divers : chercheurs, conteurs et leurs publics, agents transmetteurs de toutes sortes (professionnels ou occasionnels) captivés par la force du verbe, sans recours au texte écrit. En ce sens, l'oralité se distingue de 
l'oralisation, elle désigne une texture incarnée, infixée, négociée in vivo entre celui qui parle et celui qui écoute, par-delà le parler ordinaire de l'échange conversationnel, " œuvre performée ", selon la définition de Paul Zumthor (1987). L'on s'y plait et l'on s'y instruit. Nous la qualifierons donc de littéraire. L'art de la parole relève de l'ancienne rhétorique et, par-delà l'Antiquité où elle a d'illustres défenseurs, déjoue le piège des origines, comme les prévisions de sa disparition annoncée avec la fin des terroirs. Elle résiste à l'usure du temps au point de renaître sans cesse dans la voix singulière des conteurs, qui inventent des formes de socialité impulsées aujourd'hui par le numérique. Si certains peuvent encore la soupçonner de quelques attaches au primitivisme, après avoir servi de véhicule à bien des théories évolutionnistes et diffusionnistes, elle accompagne aujourd'hui derechef les débats sur les dynamiques des sociétés et leurs nouveaux types d'approches, disponible pour de nouvelles aventures artistiques et scientifiques.

La science en naît au XIX ${ }^{\mathrm{e}}$ siècle avec l'entreprise féconde des frères Grimm (sur le mode du collectage savant), pour se développer ensuite sous les traits du folklore et à présent du cyberlore, mot emprunté à Brunilde Biebuick (2000). C'est dans les années 1970-1980 qu'est apparu en France et dans le monde euro-américain, un phénomène qui a dépassé toutes les espérances, un renouveau, artisanal s'il en est, qui bouleverse jusqu'aux pratiques de la communication politique. Storytelling comme " machine à formater les esprits " côté américain (Salmon :2007), le contage en France s'implante dans les villes, en étroite liaison avec des dispositifs qui ont pour but de fabriquer du lien social, d'opérer des médiations culturelles entre les gens, entre les cultures en présence, entre les diverses scènes de l'éducation (qu'il s'agisse de la maîtrise de la langue ou des valeurs citoyennes). De l'interculturalisme préconisé naguère à la diversité culturelle revendiquée aujourd'hui, le conte traverse les politiques et survit aux instrumentalisations les mieux orchestrées, sans pour autant avoir acquis la reconnaissance d'une pleine légitimité. Sous cette étroite appellation générique, il reste encore pour beaucoup (recherche et sens commun sont ici à conjoindre) le domaine réservé de l'enfance, même si les nouveaux conteurs s'évertuent à conquérir des publics d'adultes. Entendons ici par « conte » l'ensemble mouvant des genres d'une littérature orale aux contours flous, aux usages brouillés par une histoire de France et que l'on pourrait analyser sous l'angle des migrations, de l'accueil programmé du divers.

Cependant il est des expressions artistiques qui mélangent les formes et génèrent autant de laboratoires offerts à la recherche, à une mise en question des façons mêmes de penser, de créer, de chercher. Il s'agira d'en présenter quelques exemples qui donneront matière à approcher concrètement tant les conséquences culturelles de la mondialisation (Appadurai) que les incidences épistémologiques de ces dernières. Comment aborder l'oral aujourd'hui - ou plutôt comment les plus récents avatars de l'oralité invitent-ils à des changements de méthodes, de dispositifs, à une rupture des paradigmes?

L'hypothèse qui préside plus particulièrement à la réunion des articles de ce numéro tient à l'idée d'un étayage entre les nomadismes 
de la voix et les nouvelles technologies à l'œuvre dans la circulation de la parole et des imaginaires. Il s'agit d'interroger les catégories, de tenter de mettre à distance nos centrismes corrélés (scripto-, audio-, ethno-), mis à mal par les virtualités conjointes, interactives, voire contrastées, de la performance contée et de l'expression numérique, à entrer dans une approche critique où se mêlent, pour utiliser la terminologie de Jack Goody, les trois raisons : orale, graphique et numérique. Les langues et cultures des régions du monde dessinent ainsi des territoires imaginaires et des espaces de rencontres, où chacun peut se sentir à la fois sédentaire et nomade, où l'ici n'est pas opposé au là-bas, le centre à la périphérie, eux à nous, mais où la surprise surgit tantôt du même et tantôt de l'autre, selon des associations d'images aléatoires. En ce sens le mot " conte " serait ici plutôt à entendre comme objet-lien, objet anthropologique par excellence, médiateur d'intelligence collective, de fait comme un remarquable catalyseur de coopération qui, à la manière du ballon, circule non pas de main en main, mais de bouche à oreille, pour reprendre les termes de Pierre Lévy (1992 :128), étonnamment « pervasif » (si l'on peut annexer le mot anglais pervasive souvent utilisé, dans la culture numérique, pour désigner un imaginaire qui entrecroise de multiples formes matérielles).

Le but est de frayer des chemins à la croisée des disciplines, de veiller à l'heuristique d'un domaine qui défie résolument toute assignation à résidence (disciplinaire, identitaire, culturelle, géographique). Mouvance, variance, circulation, traduction serviront de mots-outils pour naviguer d'un article à l'autre, d'une proposition de recherche à l'autre, sous le signe d'une éthique et d'une esthétique de la relation.

Le propos inaugural appartient à Bruno de la Salle, responsable du Centre de Littérature orale (Vendôme) et fondateur reconnu du renouveau du conte en France. Bruno de la Salle non seulement a amorcé le mouvement, mais ne cesse d'y participer activement, notamment à travers l'expérimentation d'un site particulièrement inventif clio.org. Nous le remercions ici vivement de nous avoir confié une parole d'artistechercheur, à l'écoute d'un art exigeant.

Cette introduction in medias res est suivie d'une mise en perspective des « avatars de l'oralité » à laquelle se livrent Anne-Marie Dauphin et Jean Derive. Le lecteur y trouvera maints repères pour mieux comprendre les allers et venues entre l'oralité et l'écriture, et le relais apporté par les nouvelles technologies. L'article esquisse une méthodologie novatrice qui pourra servir de guide à l'élaboration de productions hypermédias, encore si peu nombreuses dans un domaine où elles apparaissent pourtant indispensables. De nouveaux chantiers se profilent, où chacun pourra conjuguer librement les préfixes et passer de l'inter- à l'hyper, via le cyber-.

Raymond Mayer, quant à lui, revisite les notions qui ont marqué l'histoire de la recherche et lui ont fourni une sorte de charpente tout aussi rassurante que floue, souvent utilisée sans grande clarté terminologique : oralité primaire (des sociétés dites sans écriture), oralité secondaire (des sociétés - cas le plus fréquent - où l'oralité coïncide avec une pratique de l'écrit). Peut-on aujourd'hui parler d'oralité tertiaire (des 
sociétés numériques) pour mieux dépasser les binarismes où s'enlisent trop souvent les débats (oral/écrit; populaire/savant; traditionnel/ moderne; barbare/civilisé ; concret/abstrait...)?

Par-delà les théories qu'elle a pu inspirer, l'une des forces de l'oralité littéraire en ses divers avatars, réside dans sa capacité à offrir des objets concrets qui résistent à toutes formes de dogmatisme, véritables bijoux considérés par les frères Grimm comme des fragments précieux de mythes engloutis. Échappées aux greniers-herbiers de la mémoire, des paroles se déplacent, transforment les répertoires, permettent d'analyser la variation in vivo, de trouver ou créer des situations expérimentales impensables encore il y a peu d'années.

Trois exemples sont ici apportés. Le premier concerne la circulation numérique d'un chef-d'œuvre de la littérature arabe et de la littérature mondiale, si longtemps méprisé, et toujours objet de suspicion et de débat : les Mille et une Nuits. Katia Zakharia, dans son étude des sites dévolus à leur diffusion, montre comment la libre parole des conteurs à travers la figure de Shéhérazade s'inscrit, encore et toujours, dans le registre subversif du politique. Le deuxième exemple nous conduit dans le Nord-Est brésilien, où la littérature de cordel, prise entre l'oralité et écriture, entre dans une nouvelle ère numérique qui renouvelle les perspectives d'étude. Martine Kunz nous fait passer de l'in-quarto au cordel numérique, montrant comment une même matrice narrative s'accommode des mélanges. Le troisième exemple, proposé par Nadine Decourt, est, lui aussi, saisi dans une dimension diachronique et synchronique, mettant en scène un personnage qui fait figure de héros voyageur, sous le signe de la blague et de la facétie : Nasreddin Hodja (turc), alias Djoha (Maghreb), Goha (Égypte), etc. Nasreddin prend l'étoffe d'un passeur de mondes, d'un opérateur de connivences hic et nunc. L'on se demandera cependant comment se fabrique une culture commune, dans quelles limites de partage et d'opacité, par-delà l'apparente accessibilité d'un corpus en ligne?

Ce dernier exemple introduit une notion nouvelle dans les horizons de recherche (Finnegan, $2007: 196-200$ ), celle de collaborations ouvertes aux divers protagonistes des opérations de recherche, sans dominant ni dominé, notamment entre chercheurs et conteurs - partenariat que l'on pourrait qualifier ici de créatif. L'axe de recherche s'articule autour d'une poétique expérimentale de la rencontre. Ainsi Lucille Guilbert exposet-elle un dispositif d'Atelier interculturel de l'imaginaire où le conte sert d'objet médiateur, entre la fiction et le récit de vie. Le dispositif a été expérimenté avec des réfugiés au Québec, mais aussi dans le cadre d'un échange entre étudiants canadiens et étudiants chinois. Par-delà tout enfermement dans la notion d'aire culturelle, Emmanuelle Saucourt présente, quant à elle, une démarche qui participe à la fois de la recherche et de l'intervention sociale, à travers la mise à l'essai d'une forme de conférence contée où un dialogue in situ s'improvise entre conteur et chercheur.

Ainsi le dernier mot revient-il à l'exercice de la vive voix, dans l'énigme plénière de la présence et de l'écoute, selon une logique d'hybridation qui attire de plus en plus l'attention des créateurs de sites. Nous 
assumons donc le paradoxe d'un support écrit, qui porte trace et fait tremplin. Le présent numéro ne saurait se lire sans la conscience de tous les liens possibles. L'ambition n'est pas de supprimer l'écrit au profit d'un tout-numérique aussi illusoire qu'utopiste, mais bien de mettre en relation les différentes approches, au gré des opportunités et des ressources, sur un mode exploratoire ouvert à d'autres développements à venir.

\section{Références bibliographiques}

Appadurai A., Après le colonialisme. Les conséquences culturelles de la globalisation, Paris, Payot, 2001 [Modernity at Large. Cultural Dimensions of Globalization, 1996].

Biebuick B., Cahiers de Littérature orale, n 47 : p@aroles 2000.clo, « Du folklore au cyberlore. Paroles électroniques, avez-vous donc une âme?", p. 43-71.

Breton P., L'éloge de la parole, Paris, La Découverte, 2007.

Finnegan R., The oral and beyond. Doing Things with Words in Africa, James Currey Oxford, The University of Chicago Press, University of KwaZulu-Natal Press Pietermaritzburg, 2007.

Goody J., La raison graphique, Paris, Éd. de Minuit, 1979.

Hermès 39. Critique de la raison numérique, Paris, cNRs Éditions, 2004.

LÉvy P., Qu'est-ce que le virtuel?, La Découverte/Poche, 1998.

SALMON C., Storytelling, la machine à fabriquer des histoires et à formater les esprits, Paris, La Découverte, 2007.

Waguet F., Parler comme un livre. L'oralité et le savoir, Paris, Albin Michel, 2003.

Zumthor P., La lettre et la voix, Paris, Seuil, 1987. 